\title{
Microfluidic Fabrication of Stable Gas-Filled Microcapsules for Acoustic Contrast Enhancement
}

\author{
Alireza Abbaspourrad, ${ }^{\dagger}$ Wynter J. Duncanson, ${ }^{\dagger, \mathbb{I}}$ Natalia Lebedeva, ${ }^{\ddagger}$ Shin-Hyun Kim, ${ }^{\dagger, \S}$ \\ Aleksandr P. Zhushma, ${ }^{\ddagger}$ Sujit S. Datta, ${ }^{\dagger}$ Paul A. Dayton, ${ }^{\perp}$ Sergei S. Sheiko, ${ }^{\ddagger}$ Michael Rubinstein, ${ }^{\ddagger}$ \\ and David A. Weitz ${ }^{* \dagger}$ \\ ${ }^{\dagger}$ School of Engineering and Applied Sciences and Department of Physics, Harvard University, 29 Oxford Street, Cambridge, \\ Massachusetts 02138, United States \\ ${ }^{\ddagger}$ Department of Chemistry, University of North Carolina at Chapel Hill, UNC Chapel Hill, North Carolina 27599, United States \\ ${ }^{\S}$ Department of Chemical and Biomolecular Engineering, KAIST, Daejeon, South Korea \\ ${ }^{\perp}$ Joint Department of Biomedical Engineering, University of North Carolina at Chapel Hill and North Carolina State University, \\ Chapel Hill, North Carolina 27599, United States

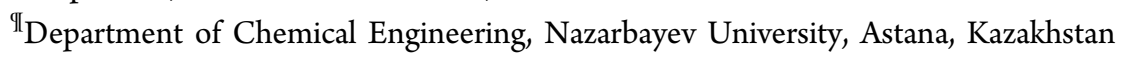

ABSTRACT: We introduce a facile approach for the production of gas-filled microcapsules designed to withstand high pressures. We exploit microfluidics to fabricate water-filled microcapsules that are then externally triggered to become gasfilled, thus making them more echogenic. In addition, the gas-filled microcapsules have a solid polymer shell making them resistant to pressure-induced buckling, which makes them more mechanically robust than traditional prestabilized microbubbles; this should increase the potential of their utility for acoustic imaging of porous media with high hydrostatic pressures such as oil reservoirs.

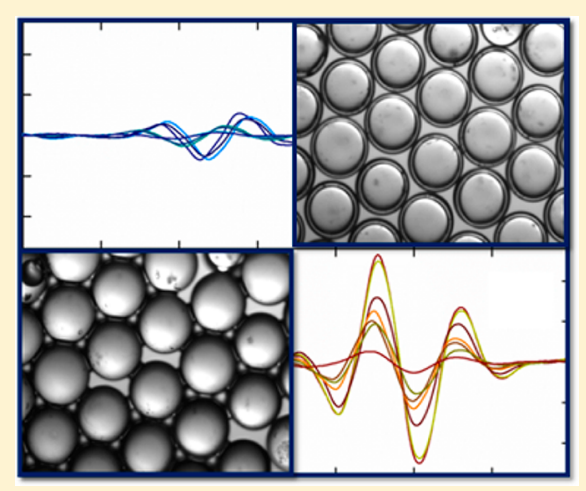

\section{INTRODUCTION}

Ultrasonic imaging is a noninvasive, long-range visualization technique in which sound waves are transmitted into and received from an object; the echoes, or the intensities of the reflected and backscattered waves, are used to form the ultrasound image. ${ }^{1}$ The extent to which a material gives rise to these reflections is its echogenicity, which is proportional to the speed of sound and the density of the reflecting material relative to that of its surroundings. For example, a solid sphere in water will generate low contrast and hence low image quality. To enhance the acoustic contrast, an object with a higher compressibility, such as a suspension of gas bubbles, is preferable. ${ }^{2,3}$ Unfortunately, gas bubbles are inherently unstable because of their rapid dissolution in liquids and resultant shortlived echogenicity. To increase their lifespan, gas bubbles can be coated with a shell that forms a protective layer and minimizes dissolution; microbubble shells composed of polymers provide the greatest stabilization., ${ }^{3,4}$ These microbubbles are easily produced by sonication; however, the uncontrolled shear of sonication yields microbubbles with broad distributions of sizes and shell thicknesses ${ }^{5-7}$ and hence a widely distributed acoustic response. Thus, the utility of these polydisperse bubbles is somewhat limited. One promising strategy for addressing this limitation is through the use of controlled synthesis strategies, such as droplet microfluidics, to produce microbubbles with precisely controlled sizes and shell thicknesses. $^{6-8}$ Such improved gas bubbles are well-suited to the demands of the most common use of ultrasonic imaging, biomedical applications. $^{9-22}$ An additional potential use of acoustic contrast agents, such as these bubbles, is that of imaging fluid components in porous media. However, this application often requires the bubbles to withstand large hydrostatic pressures ranging from 0.3 to $55 \mathrm{MPa}$; if this were feasible, then such stable microbubbles could also be used for imaging oil reservoirs. Unfortunately, most polymer-shelled microbubbles cannot withstand such enormous pressures. Moreover, because gases remain prone to dissolution even after stabilization, the application of these microbubbles is still limited. ${ }^{6,23,24}$ One means of overcoming this limitation is to use a core with a liquid placeholder that can then be externally triggered to be filled with gas on demand. ${ }^{24}$ This can increase the utility of microbubbles. Combining this core with a mechanically robust shell would enable microbubbles to be used for high-pressure acoustic imaging applications. However, microbubbles that utilize this approach have not been reported. If such bubbles were available, then they would be of particular

Received: July 15, 2013

Revised: August 30, 2013

Published: September 2, 2013 


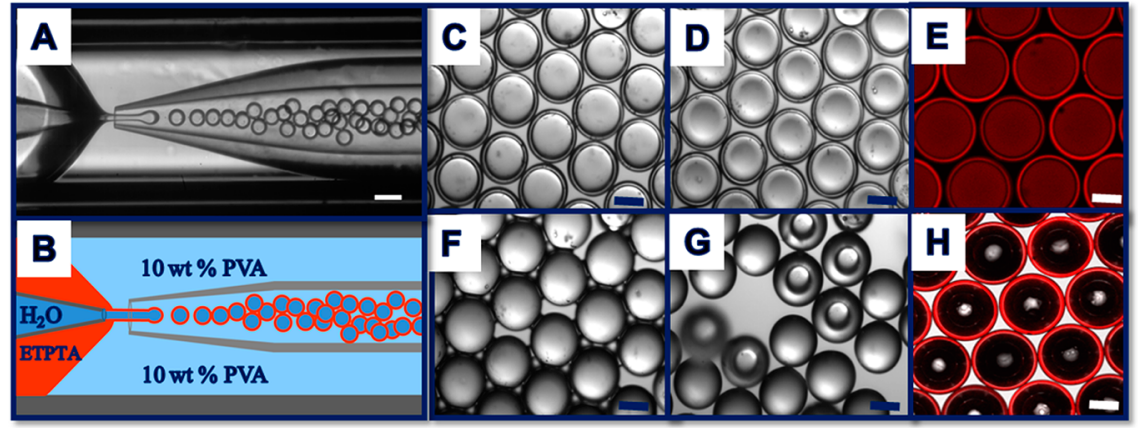

Figure 1. (A) Optical microscope image showing droplet formation in the dripping regime. The scale bar is $150 \mu \mathrm{m}$. (B) Schematic illustration of a microfluidic device for the fabrication of polymer shell double emulsion droplets. (C, D) Optical micrographs of the resulting water-filled microcapsules at different stages of thermal treatment. (E) Confocal microscope image of water-filled micocapsules showing fluorescent signals in the core. (F, G) Optical micrograph of resultant microcapsules at different stages of thermal treatment to obtain gas-filled microcapsules. (H) Confocal microscope image of gas-filled microcapsules resuspended in water. Each scale bar in C, D, F, and G is $80 \mu \mathrm{m}$, and each scale bar in $\mathrm{E}$ and $\mathrm{H}$ is 70 $\mu \mathrm{m}$.

interest for use as contrast agents in oil reservoirs in addition to their promise for use in biomedical applications.

Here, we present a technique to create robust gas-filled microcapsules using monodisperse water-filled microcapsules that are subsequently externally triggered to replace water with a gas. A glass capillary microfluidic device is used to generate water-in-oil-in-water double emulsions in which the innermost phase is water, the outermost phase is water containing a surfactant, and the middle is an oil phase containing a photocurable monomer. Upon UV irradiation, the monomer polymerizes, thereby yielding hard-shelled microcapsules that are water-filled. Subsequent drying and evaporation of the water in the core creates a large cavity and enables gas entry from the surroundings. The creation of this large cavity does not compromise the microcapsule integrity for capsules at a critical shell thickness that is experimentally determined and well described by shell theory. The resulting gas-filled microcapsules are stable under hydrostatic pressures up to 6.8 $\mathrm{MPa}$. Furthermore, these gas-filled microcapsules also provide measurable acoustic contrast at an ultrasonic frequency of 1 $\mathrm{MHz}$.

\section{RESULTS AND DISCUSSION}

We make monodisperse water-filled capsules in a glass microcapillary device. It consists of a tapered round injection and a collection capillary that are coaxially aligned within a square capillary, as shown in the bright-field image in Figure $1 \mathrm{~A}$. We fluorescently label the inner aqueous phase (5 wt \% of PVA) with rhodamine 6G (Sigma-Aldrich) and allow it to flow through the tapered round injection capillary. The middle hydrophobic phase is ethoxylated trimethylolpropane triacrylate (ETPTA, MW 457, Sigma-Aldrich) liquid monomer and a photocurable initiator (0.2 wt, \%), 2-hydroxy-2-methylpropiophenone (Daracur 1173); it flows through the region between the injection capillary and the square capillary. The outer aqueous phase, which is a $10 \%$ poly(vinyl alcohol) (PVA, MW 13 000-23 000, 87\% hydrolyzed, Sigma-Aldrich) solution, flows in the opposite direction in the space between the collection capillary and the square capillary; the resultant hydrodynamic flow-focusing leads to the formation of monodisperse double emulsions, as shown in the schematic in Figure 1B. We monitor microcapsule generation using an inverted optical microscope (DM-IRB, Leica) that is equipped with a high-speed camera (Phantom V9, Vision Research). As the double emulsions are generated, they are irradiated in situ with UV light that photopolymerizes the ETPTA monomer to form the microcapsule shell. Once the shell hardens, the microcapsules are washed to remove the PVA surfactant and then extracted by filtration and resuspended in water on a glass slide, as seen in Figure 1C. We washed the microcapsules with DI water three times to remove PVA from the continuous phase. To initiate the thermally induced core-replacement process, we hold the water-filled microcapsules at $40{ }^{\circ} \mathrm{C}$ for 20 min. As the water surrounding them evaporates, the capsules appear to indent, as shown in Figure 1D; this optical artifact is due to the lowering level of the water on the glass slide. We obtained a confocal image of capsule cross-sections; the existence of the fluorescent signal confirms that capsules are still water-filled at this stage, as shown in Figure 1E. The remaining water is drawn to the interfaces between the microcapsules by capillarity to form pendular rings at the contact points between them, as shown in Figure 1F. The water in the core of the microcapsules is replaced by air as the water fully evaporates, as seen in the image in Figure 1G. After drying, we resuspend the gas-filled microcapsules in water and use confocal microscopy to confirm that the water in the core has been replaced by the gas, as shown in Figure $1 \mathrm{H}$.

We find that the microcapsules buckle during water evaporation. ${ }^{25}$ To elucidate the origin of this behavior, we study the buckling of microcapsules having the same outer radius, $R$, but varying shell thicknesses, $t$. We produce the different shell thicknesses by varying the values of the flow rates of the inner and middle phases, $Q_{1}$ and $Q_{2}$, respectively, while keeping their sum constant at $1000 \mu \mathrm{L} / \mathrm{h}$ and while maintaining the outermost fluid flow rate at $8000 \mu \mathrm{L} / \mathrm{h}$. Images of the generation of microcapsules with different shell thicknesses are shown in Figure $2 \mathrm{~A}-\mathrm{G}$. The thicknesses are plotted as a function of the ratio of middle- to inner-phase flow rates $\left(Q_{2} /\right.$ $\left.Q_{1}\right)$ in Figure $2 \mathrm{H}$. The data are well described by a logarithmic function; this enables us to determine easily the flow rates needed to obtain microcapsules with a desired shell thickness. We use optical microscopy to probe the microcapsule buckling upon drying. The fraction of unbuckled microcapsules increases with increasing shell thickness, plateauing at a critical value of the shell thickness, approximately equal to $15 \mu \mathrm{m}$, as shown in Figure 3A. Capsules with thin shells readily buckle when dried, as shown by the first two points in Figure 3A. The buckled gasfilled microcapsules can be seen in the micrographs in Figure 

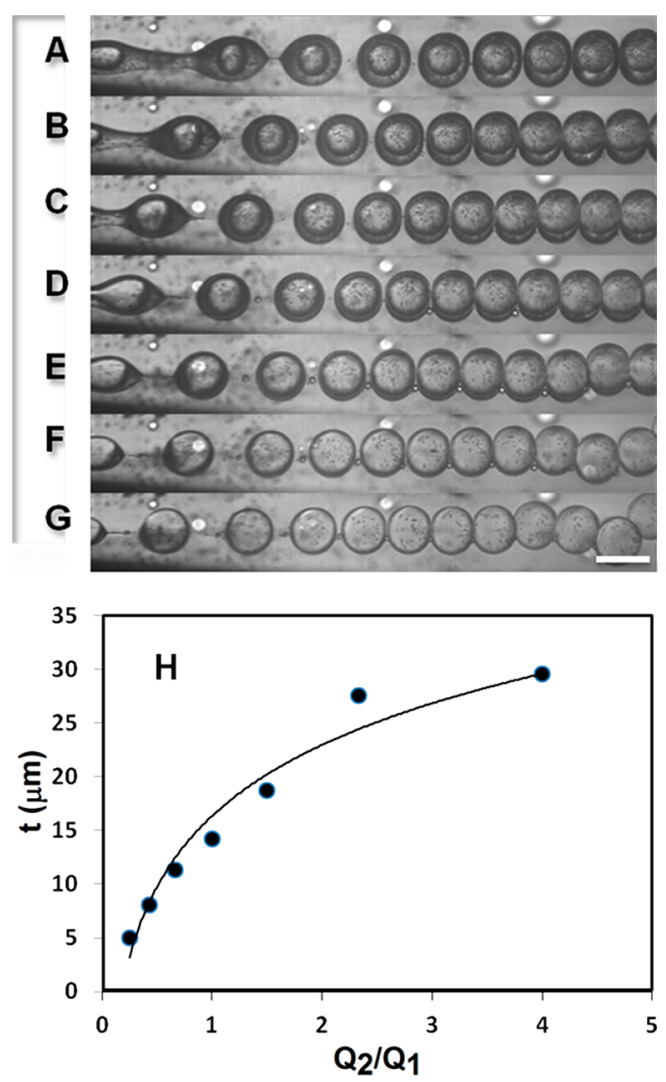

Figure 2. (A-G) Optical micrographs of double emulsions produced in the dripping regime with different shell thicknesses. $(\mathrm{H})$ Microbubble shell thicknesses are plotted as a function of the ratio of middle- to inner-phase flow rates $\left(Q_{2} / Q_{1}\right)$. The scale bar is $150 \mu \mathrm{m}$. The solid line represents the logarithmic function described in the text.

3B,C. In stark contrast, capsules with shells thicker than $\sim 0.14$ times their radius do not buckle as they dry, as shown in Figure $3 \mathrm{~A}$ and exemplified by the micrographs in Figure 3D,E. We explain this buckling threshold using shell theory: for a capsule shell to buckle, the capillary pressure difference across it, $\mathrm{P}_{c}$, must exceed a threshold $P_{\text {buckling }} \cong 2 E /\left(3\left(1-v^{2}\right)\right)^{1 / 2}(t / R)^{2}$, where $E \approx 600 \mathrm{MPa}$ and $\nu \approx 1 / 3$ are the Young's modulus and Poisson ratio of the shell material, respectively, ${ }^{26}$ and $R$ is the shell radius. ${ }^{27}$ As shown experimentally, the onset of buckling occurs at $t / R=0.14$; this corresponds to a threshold pressure of $P_{\text {buckling }} \approx 14 \mathrm{MPa}$. We hypothesize that, as the water evaporates, tiny menisci form in the pores of the cross-linked polymer microcapsule shells. This results in a capillary pressure difference across each shell; the pressure outside is greater than that inside by an amount $P_{\mathrm{c}} \approx 2 \gamma / a_{\mathrm{p}}$, where $\gamma \approx 70 \mathrm{mN} / \mathrm{m}$ is the surface tension of water in air at $40{ }^{\circ} \mathrm{C}$ and $a_{\mathrm{p}}$ is the typical radius of a pore. Balancing the buckling pressure with the capillary pressure, $P_{\mathcal{c}}$ yields a characteristic pore radius of $a_{\mathrm{p}} \approx$ $10 \mathrm{~nm}$, consistent with that expected for a highly cross-linked polymer shell. These results thus indicate that only capsules with sufficiently thick shells are stable against buckling during water evaporation.

To extend the utility of these capsules for applications that require them to operate under high hydrostatic pressures, such as in oil reservoirs, they must withstand hydrostatic pressures that range between 6.8 and $55.1 \mathrm{MPa}$. To investigate the behavior of our polymer-shelled microcapsules under such high pressures, we designed a high-pressure cell with imaging capabilities using one piece of 316 stainless steel with a sapphire window and one solid piece; these are attached using screwtight flanges. Steel tubing was connected to two ports along the circumference of the steel cylinder for liquid flow through the cell. Pressure is generated using an automated syringe pump (ISCO model 260D) rated to 51.7 MPa. Temperature control of the cell is maintained using an autotune unit (Omega CN77353-A2) with a K-type thermocouple and an Omega flexible heater (silicon rubber) covered with glass wool insulating tape. The pressure inside the cell is monitored using an output pressure transducer (model TJE/743-11TJG, Sensotec, Columbus, OH) and gauges (Sensotec, type GM). Microcapsules inside the cell are observed by optical microscopy through transparent capping sapphire windows. The imaging system consisted of a 12 megapixel (Canon 450D) camera with a EF-S $18-55 \mathrm{~mm}, \mathrm{f} / 3.5-5.6$ lens. Static images and videos of the gas-filled microcapsules were acquired under various pressures ranging from 1 to $490 \mathrm{~atm}(0.1$ to $50 \mathrm{MPa})$. Using this high-pressure imaging cell allows simultaneous pressurization and visualization of individual gas-filled microcapsules. At lower pressures (e.g., $5.5 \mathrm{MPa}$ ), all capsules remain intact, as seen in Figure 4A. When the pressure reaches 11.7 $\mathrm{MPa}$, we observe that the initially dark core of one of the capsules in Figure 4B becomes lighter as a result of water penetrating the capsule through a microscopic pore in its shell. We observe similar behavior at $22-27 \mathrm{MPa}$ for two other bubbles, as shown in Figure $4 \mathrm{D}-\mathrm{H}$. We believe that this variance in the shell-fracture pressure reflects the corresponding

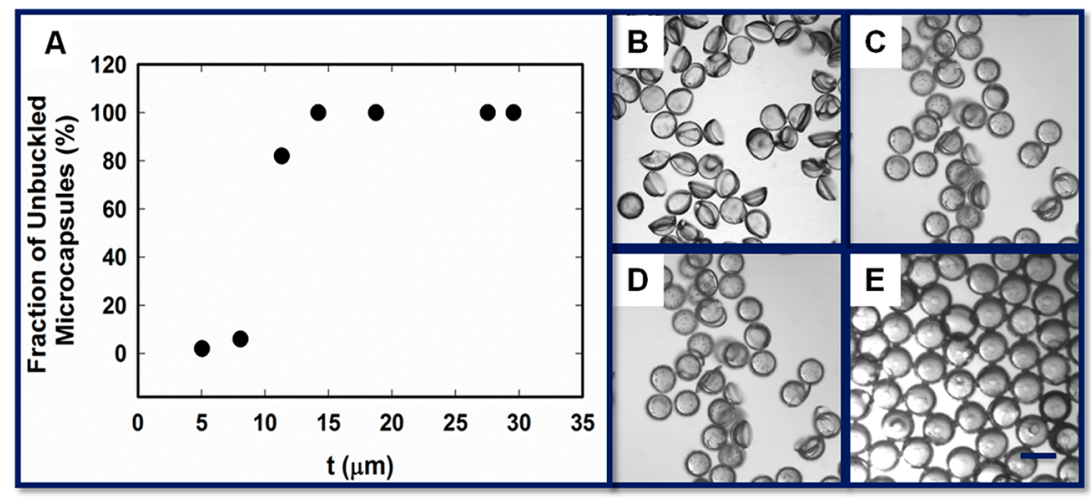

Figure 3. (A) Fraction of unbuckled gas-filled microcapsules with different shell thicknesses. (B-E) Optical microscope images of dried microcapsules. The scale bar is $150 \mu \mathrm{m}$. 


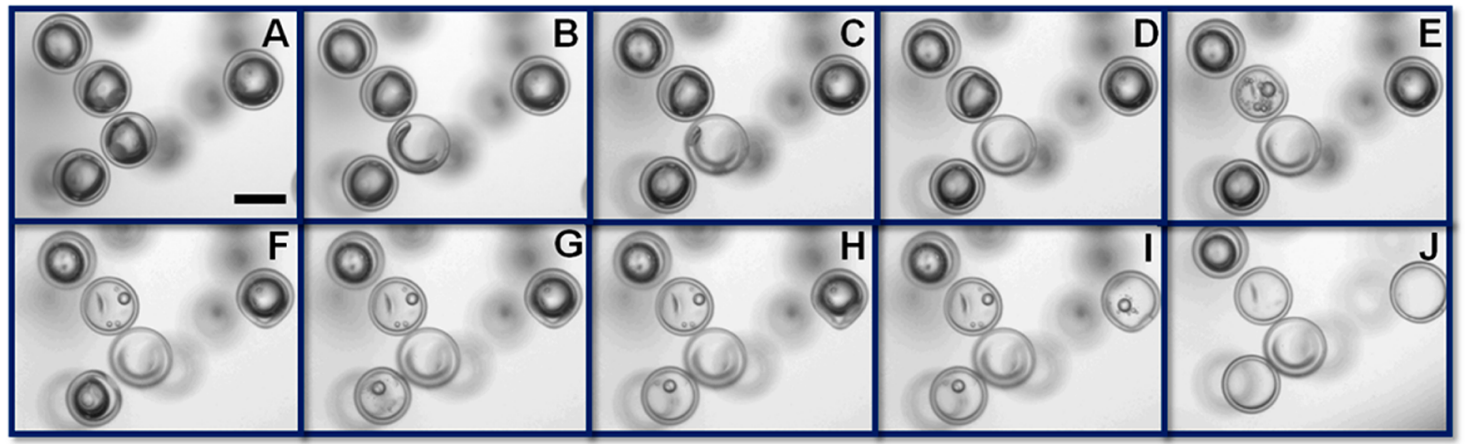

Figure 4. (A-J) Gas-filled microcapsules subjected to high hydrostatic pressure up to $50 \mathrm{MPa}$. The scale bar is $120 \mu \mathrm{m}$.

variance in the gas-filled microcapsule shell thicknesses. At even higher pressures, water continues to infiltrate the shell and forces the gas to dissolve fully in the water, as shown in Figures 4I,J. To understand this behavior, we analyze the distribution of pressures in the fluids: we hypothesize that as the water surrounding a shell tries to squeeze through it, tiny menisci form in the pores. This results in a capillary pressure difference across the shell; the pressure inside the shell is greater by $P_{\mathrm{c}}=$ $2 \gamma / a_{\mathrm{p}}$, where $\gamma \approx 70 \mathrm{mN} / \mathrm{m}$ is again the surface tension between air and water. Thus, to begin to fill the capsule interior, the hydrostatic pressure applied to the water, $P_{\mathrm{h}}$, must exceed a threshold given by $P_{c}{ }^{28}$ balancing the observed threshold $P_{\mathrm{h}} \approx$ 11.7 MPa with $P_{\mathrm{c}}$ yields an independent estimate of the typical pore radius, $a_{\mathrm{p}} \approx 12 \mathrm{~nm}$. This is in excellent agreement with the estimate of $a_{\mathrm{p}}$ from our observations of the gas-filled microcapsule buckling. Conversely, to prevent water-infiltration, $P_{\mathrm{h}}$ must be less than $P_{\mathrm{c}}$; this criterion provides a key requirement for producing gas-filled microcapsules that are able to withstand reservoir pressures.

To evaluate the utility of these gas-filled microcapsules as acoustic contrast agents, we visualize and insonate them. We carry out acoustic detection under water submersion using a custom-built polycarbonate chamber. The microcapsule sample holder is constructed as a fixed-volume chamber (approximately $750 \mu \mathrm{L}$ ) with two layers of optically and acoustically transparent plastic sheets that are separated by a rubber gasket. A $0.75 \mathrm{in}$. diameter, $1 \mathrm{MHz}$ immersion transducer with a $-6 \mathrm{~dB}$ beam width at its focus, spherically focused at $40 \mathrm{~mm}$ (Olympus NTD), is aligned with the sample holder and coaxially aligned with the camera. The $-6 \mathrm{~dB}$ beam width at the transducer focus is approximately $3.2 \mathrm{~mm}$. A single sine wave pulse $(1 \mathrm{MHz}, 400 \mathrm{~V})$ with a repetition rate of $100 \mathrm{~Hz}$ is generated by a pulse generator/receiver (Olympus 5077PR). The peak pressure at the acoustic focus is $17 \mathrm{kPa}$ with a calibrated needle hydrophone (HNC-0400, Onda Corp). The resulting echo is reflected back to the transducer and digitized with an oscilloscope (LeCroy 9314AM) at $100 \mathrm{MHz}$ and transferred to a PC. Using LabView, 100 scans are averaged for each concentration of microcapsules. The microcapsule concentration is quantified by counting the number of capsules in the acoustic focus $(3.2 \mathrm{~mm})$ of the transducer. The small acoustic reflection of the plastic windows in the holder is subtracted from each data set. The peak amplitude of the echo reflected from the microcapsule layer is measured as a function of microcapsule concentration. We investigate the impact of microcapsule concentration on echogenicity by testing seven concentrations. The microcapsules form a submonolayer, as shown for three concentrations in Figure 5A-C. To determine
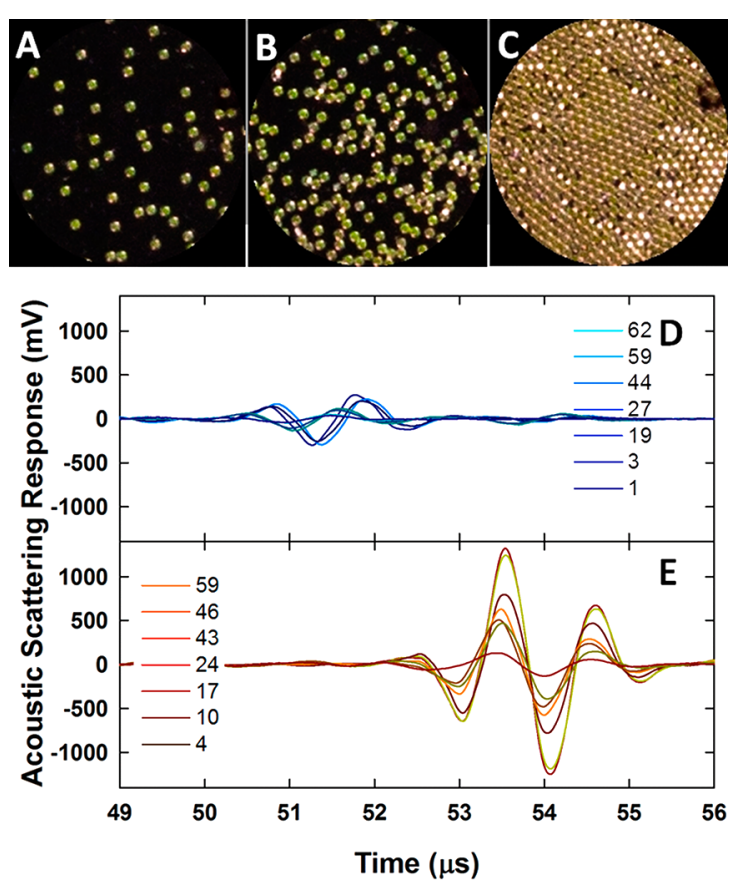

Figure 5. (A-C) Optical micrograph of three different concentrations of gas-filled microcapsules. (D) Acoustic scattering response of the water-filled microcapsules. (E) Acoustic scattering response of the gasfilled microcapsules. Numbers with different colors represent the number of capsules in the acoustic focal plane.

the effective echogenicity of the gas-filled microcapsules, we compare them to water-filled microcapsules. The echoes from the gas-filled capsules occur later than those of the water-filled capsules, because their greater buoyancy positions them further from the transducer. In addition, the amplitude of the acoustic scattering response for both the water-filled and the gas-filled microcapsules increases with concentration, as shown in Figures 5D,E, respectively. However, the amplitudes from the gas-filled microcapsules are consistently greater than those from an identical concentration of water-filled microcapsules; hence, we conclude that the gas-filled microcapsules are more echogenic than the water-filled microcapsules. ${ }^{14}$ To quantify this, we plot the peak amplitude of the scattering response as a function of concentration; the scattering exhibits a linear increase with concentration, as shown by the solid lines through the data in Figure 6. Echogenicity is proportional to the value of the slope and is $3.03 \mathrm{mV} / \mathrm{mm}^{2}$ for the water-filled microcapsules and $19.9 \mathrm{mV} / \mathrm{mm}^{2}$ for the gas-filled microcapsules; this demonstrates that the difference in echogenicity is due 


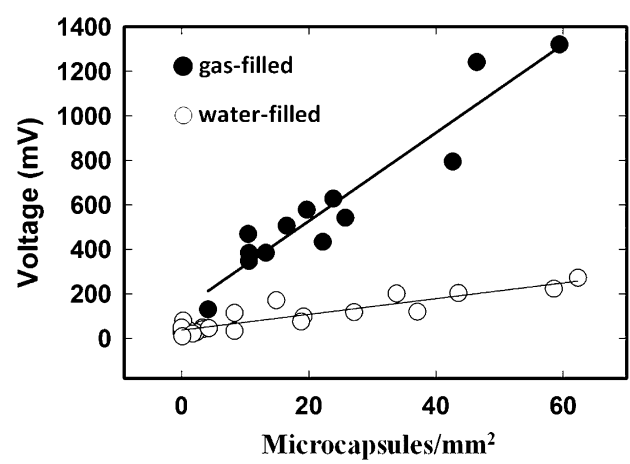

Figure 6. Scattering response of gas-filled and water-filled microcapsules as a function of microcapsule concentration.

solely to the composition of the microcapsule core. Such gasfilled microcapsules can be useful for enhancing echogenicity in high-pressure applications.

\section{CONCLUSIONS}

Gas-filled microcapsules that are formed by traditional strategies are limited in use to biomedical acoustic imaging because they are not designed to withstand high pressures; hence, they cannot be used in the acoustic imaging of oil reservoirs. The strategy introduced here addresses these limitations through the use of microfluidics to create waterfilled microcapsules that are then externally triggered to become gas-filled, thus making them more echogenic. ${ }^{14}$ In addition, the microcapsules have a solid polymer shell, making them resistant to pressure-induced buckling, which makes them more mechanically robust than traditional prestabilized gasfilled microparticles; this should increase the potential of their utility for acoustic imaging of oil reservoirs. Moreover, the use of microfluidics enables the direct incorporation of additional components, such as magnetic nanoparticles, into the shell; ${ }^{29}$ this allows the microcapsules to be directed to specific locations by an externally applied magnetic field and may enhance the stability of the shell. ${ }^{30,31}$ Therefore, these microcapsules also have great potential in targeted ultrasonic imaging applications. We note that such applications often require microcapsule diameters in the range of $\sim 1-10 \mu \mathrm{m}$, significantly smaller than the microcapsules we use here; reducing the microcapsule size would thus be an interesting direction for future work.

\section{AUTHOR INFORMATION}

\section{Corresponding Author}

*E-mail: weitz@seas.harvard.edu.

\section{Author Contributions}

A.A. and W.J.D. contributed equally to this work.

\section{Notes}

The authors declare no competing financial interest.

\section{ACKNOWLEDGMENTS}

This work was supported by the Advanced Energy Consortium (http://www.beg.utexas.edu/aec/), whose member companies include BP America Inc., BG Group, Petrobras, Schlumberger, Shell, and Total. We thank Rachel Miller and Thomas E. Kodger for their critical reviews of this manuscript. S.S.D. acknowledges support from ConocoPhillips.

\section{REFERENCES}

(1) Foster, F. S.; Pavlin, C. J.; Harasiewicz, K. A.; Christopher, D. A.; Turnbull, D. H. Advances in Ultrasound Biomicroscopy. Ultrasound Med. Biol. 2000, 26, 1-27.

(2) Ophir, J.; Parker, K. J. Contrast Agents in Diagnostic Ultrasound. Ultrasound Med. Biol. 1989, 15, 319-333.

(3) Ferrara, K.; Pollard, R.; Borden, M. Ultrasound Microbubble Contrast Agents: Fundamentals and Application to Gene and Drug Delivery. Annu. Rev. Biomed. Eng. 2007, 9, 415-447.

(4) Duncanson, W. J.; Oum, K.; Eisenbrey, J. R.; Cleveland, R. O.; Wheatley, M. A.; Won, J. Y. Targeted Binding of PEG-Lipid Modified Polymer Ultrasound Contrast Agents with Tiered Surface Architecture. Biotechnol. Bioeng. 2010, 106, 501-506.

(5) Xu, J. H.; Chen, R.; Wang, Y. D.; Luo, G. S. Controllable Gas/ Liquid/Liquid Double Emulsions in a Dual-Coaxial Microfluidic Device. Lab Chip 2012, 12, 2029-2036.

(6) Lee, M. H.; Lee, D. Elastic Instability of Polymer-Shelled Bubbles Formed from Air-in-Oil-in-Water Compound Bubbles. Soft Matter 2010, 6, 4326-4330.

(7) Parhizkar, M.; Edirisinghe, M.; Stride, E. Effect of Operating Conditions and Liquid Physical Properties on the Size of Monodisperse Microbubbles Produced in a Capillary Embedded TJunction Device. Microfluid. Nanofluid. 2013, 14, 797-808.

(8) Gunduz, O.; Ahmad, Z.; Stride, E.; Tamerler, C.; Edirisinghe, M. Bioinspired Bubble Design for Particle Generation. J. R. Soc. Interface 2012, 9, 389-395.

(9) Fiddesa, L. K.; Raza, N.; Srigunapalan, S.; Tumarkana, E.; Simmons, C. A.; Wheelera, A. R.; Kumacheva, E. A Circular CrossSection PDMS Microfluidics System for Replication of Cardiovascular Flow Conditions. Biomaterials 2010, 31, 3459-3464.

(10) Park, J. I.; Jagadeesan, D.; Williams, R.; Oakden, W.; Chung, S.; Stanisz, G. J.; Kumacheva, E. Microbubbles Loaded with Nanoparticles: A Route to Multiple Imaging Modalities. ACS Nano 2010, 4, 6579-6586.

(11) Pancholi, K. P.; Farook, U.; Moaleji, R.; Stride, E.; Edirisinghe, M. J. Novel Methods for Preparing Phospholipid Coated Microbubbles. Eur. Biophys. J. 2008, 37, 515-520.

(12) Stride, E.; Edirisinghe, M. Novel Preparation Techniques for Controlling Microbubble Uniformity: A Comparison. Med. Biol. Eng. Comput. 2009, 47, 883-892.

(13) Stride, E.; Edirisinghe, M. Novel Microbubble Preparation Technologies. Soft Matter 2008, 4, 2350-2359.

(14) Azmin, M.; Harfield, C.; Ahmad, Z.; Edirisinghe, M.; Stride, E. How Do Microbubbles and Ultrasound Interact? Basic Physical, Dynamic and Engineering Principles. Curr. Pharm. Des. 2012, 18, 2118-2134.

(15) Zhang, H.; Tumarkin, E.; Peerani, R.; Nie, Z.; Sullan, R. M. A.; Walker, G. C.; Kumacheva, E. Microfluidic Production of Biopolymer Microcapsules with Controlled Morphology. J. Am. Chem. Soc. 2006, $128,12205-12210$.

(16) Stride, E. The Influence of Surface Adsorption on Microbubble Dynamics. Philos. Trans. R. Soc. A 2008, 366, 2103-2115.

(17) Hettiarachchi, K.; Lee, A. P. Polymer-Lipid Microbubbles for Biosensing and the Formation of Porous Structures. J. Colloid Interface Sci. 2010, 344, 521-527.

(18) Farook, U.; Stride, E.; Edirisinghe, M. J. Preparation of Suspensions of Phospholipid-Coated Microbubbles by Coaxial Electrohydrodynamic Atomization. J. R. Soc., Interface 2009, 6, 271277.

(19) Zhou, M.; Cavalieri, F.; Caruso, F.; Ashokkumar, M. Confinement of Acoustic Cavitation for the Synthesis of ProteinShelled Nanobubbles for Diagnostics and Nucleic Acid Delivery. ACS Macro Lett. 2012, 1, 853-856.

(20) Cai, X.; Yang, F.; Gu, N. Applications of Magnetic Microbubbles for Theranostics. Theranostics 2012, 2, 103-112.

(21) Daiguji, H.; Takada, S.; Cornejo, J. J. M.; Takemura, F. Fabrication of Hollow Poly(lactic acid) Microcapsules from Microbubble Templates. J. Phys. Chem. B 2009, 113, 15002-15009. 
(22) Park, J. I.; Nie, Z.; Kumachev, A.; Abdelrahman, A. I.; Binks, B. P.; Stone, H. A.; Kumacheva, E. A Microfluidic Approach to Chemically Driven Assembly of Colloidal Particles at Gas-Liquid Interfaces. Angew. Chem. 2009, 121, 5404-5408.

(23) Lee, M. H.; Prasad, V.; Lee, D. Microfluidic Fabrication of Stable Nanoparticle-Shelled Bubbles. Langmuir 2010, 26, 2227-2230.

(24) Duncanson, W. J.; Abbaspourrad, A.; Shum, H. C.; Kim, S. H.; Adams, L. L.; Weitz, D. A. Monodisperse Gas-Filled Microparticles from Reactions in Double Emulsions. Langmuir 2012, 28, 6742-6745.

(25) Tsapis, N.; Dufresne, E. R.; Sinha, S. S.; Riera, C. S.; Hutchinson, J. W.; Mahadevan, L.; Weitz, D. A. Onset of Buckling in Drying Droplets of Colloidal Suspensions. Phys. Rev. Lett. 2005, 94, 018302-4.

(26) Lin, T. H.; Huang, W. H.; Jun, I. K.; Jiang, P. Bioinspired Assembly of Colloidal Nanoplatelets by Electric Field. Chem. Mater. 2009, 21, 2039-2044.

(27) Datta, S. S.; Kim, S. H.; Paulose, J.; Abbaspourrad, A.; Nelson, D. R.; Weitz, D. A. How Does a Porous Shell Collapse? Delayed Buckling and Guided Folding of Inhomogeneous Capsules. Phys. Rev. Lett. 2012, 109, 134302-134305.

(28) Krummel, A. T.; Datta, S. S.; Munster, S.; Weitz, D. A. Visualizing Multiphase Flow and Trapped Fluid Configurations in a Model Three-Dimensional Porous Medium. AIChE J. 2013, 59, 10221029.

(29) Gong, X.; Peng, S.; Wen, W.; Sheng, P.; Li, W. Design and Fabrication of Magnetically Functionalized Core/Shell Microspheres for Smart Drug Delivery. Adv. Funct. Mater. 2009, 19, 292-297.

(30) Mohamedi, G.; Azmin, M.; Pastoriza-Santos, I.; Huang, V.; Pérez-Juste, J.; Liz-Marzan, L. M.; Edirisinghe, M.; Stride, E. Effects of Gold Nanoparticles on the Stability of Microbubbles. Langmuir 2012, 28, 13808-13815.

(31) Azmin, M.; Mohamedi, G.; Edirisinghe, M.; Stride, E. Dissolution of Coated Microbubbles: The Effect of Nanoparticles and Surfactant Concentration. Mater. Sci. Eng. 2012, 13, 2654-2658. 